\title{
Hábito alimentar de Alouatta guariba (Humboldt) (Primates, Atelidae) em Floresta de Araucária, Paraná, Brasil ${ }^{1}$
}

\author{
João M. D. Miranda ${ }^{2} \&$ Fernando C. Passos ${ }^{3}$ \\ 1 Contribuição número 1530 do Departamento de Zoologia, Universidade Federal do Paraná. \\ ${ }^{2}$ Rua Rio Guaporé 1275, Bairro Alto, 82840-320 Curitiba, Paraná. E-mail: guaribajoao@yahoo.com.br \\ ${ }^{3}$ Departamento de Zoologia, Universidade Federal do Paraná. Caixa Postal 19020, 81531-980 Curitiba, Paraná, Brasil. \\ Bolsista do CNPq. E-mail: fpassos@ufpr.br
}

\begin{abstract}
Feeding habits of the Alouatta guariba (Humboldt) (Primates, Atelidae) on a Araucaria Pine Forest, Paraná, Brazil. This work was made between February 2002 and January 2003, on Payquerê Farm, localized on Balsa Nova Municipality, Paraná State, Brazil. The objects were: to quantify the time employee by $A$. guariba (Humboldt, 1812) eating leaves, fruits and flowers; as well as know the species of the plants used in Brown Howler monkey feeding, in a natural place of a Araucaria Pine Forest. In an all time used in feeding, the Bugre's Howlers employed: $57 \%$ eating leaves, $41 \%$ fruits and $1,7 \%$ flowers. The Howler Monkeys feeding activity were observed in 70 trees, belonging to 34 species in 19 families, including an expressive number of pioneer species and two exotic species. These results suggest a good capability of this primate to be adapted in a partial changed place and live in new places.

KEY WORDS. Brown howler monkey, feeding behavior, primate ecology.
\end{abstract}

RESUMO. Este trabalho foi feito entre fevereiro de 2002 e janeiro de 2003, na Chácara Payquerê, localizada no município de Balsa Nova, Estado do Paraná, Brasil. Os objetivos foram: quantificar o tempo empregado por $A$. guariba (Humboldt, 1812) comendo folhas, frutos e flores; bem como conhecer as espécies vegetais utilizadas na dieta dos bugios ruivos, em ambiente natural de Floresta de Araucária. De todo o tempo utilizado na alimentação, os bugios do Bugre empregaram: $57 \%$ comendo folhas, $41 \%$ frutos e 1,7\% flores. Os bugios foram observados se alimentando em 70 árvores, pertencendo a 34 espécies em 19 famílias, incluindo um número expressivo de espécies pioneiras e duas espécies exóticas. Estes resultados sugerem uma boa capacidade deste primata em se adaptar a um ambiente parcialmente alterado e colonizar novos ambientes.

PALAVRAS CHAVE. Bugio-ruivo, comportamento alimentar, ecologia de primatas.

Os primatas do gênero Alouatta Lacèpéde, 1799 são os mais folívoros dentre os macacos neotropicais e são, como um todo, chamados de folívoros comportamentais, tendo a necessidade de descansar uma grande parte do dia, facilitando assim a digestão (Milton 1980, Strier 1992, Auricchio 1995, Bicca-MarQues \& Calegaro-Marques 1995).

No Estado do Paraná, Sul do Brasil, ocorrem duas espécies do gênero Alouatta: A. caraya (Humboldt, 1812) e A. guariba (Humboldt, 1812). A primeira, Alouatta caraya (bugio-preto) tem ocorrência limitada ao extremo Oeste e Noroeste do Estado. A segunda, Alouatta guariba (bugio-ruivo), apresenta-se distribuída por todo o Paraná, associada à cobertura florestal. $\mathrm{O}$ bugio-ruivo ocupa o Paraná desde o Leste acompanhando a Floresta Ombrófila Densa, passando pela Floresta Ombrófila Mista ( = Floresta de Araucária) entrando no Primeiro e Segundo Planaltos paranaenses e chegando ao Oeste do Estado na
Floresta Estacional Semidecidual. Em parte desta última formação florestal ocorre uma zona de simpatria entre as duas espécies de bugios (Cabrera 1957, Lange \& Jablonski 1981, Auricchio 1995, Emmons \& Feer 1997, Nowak 1999, RedFord \& EISENBERG 1999).

Hoje, no entanto, a Floresta de Araucária encontra-se altamente fragmentada, restringindo assim a presença de populações representativas do bugio-ruivo neste ambiente, a grandes fragmentos-refúgios. Exemplos destes remanescentes florestais são os do centro-sul do Paraná (Município de General Carneiro) e os encontrados no sopé da Escarpa Devoniana, passando pelos Municípios de Campo Largo e Balsa Nova. Devido ao alto grau de fragmentação em que a cobertura vegetal do Paraná atualmente se encontra é que o A. guariba foi colocado na categoria vulnerável de ameaça de extinção para o Estado (Margarido \& Lange 1995, Margarido \& Braga 2004).

Revista Brasileira de Zoologia 21 (4): 821-826, dezembro 200W 
As espécies vegetais que caracterizam a Floresta de Araucária são predominantemente diferentes das espécies contidas na Floresta Ombrófila Densa e das espécies da Floresta Estacional Semidecidual (MaAck 1968, Ibge 1992, Roderjan et al. 1998). Assim sendo, torna-se de grande valor científico, ecológico e conservacionista conhecer as espécies utilizadas na alimentação do bugio-ruivo, bem como entender como se dá a exploração dos recursos alimentares dentro do bioma Floresta de Araucária.

\section{MATERIAL E MÉTODOS}

\section{Área de estudo}

O presente estudo foi realizado na Chácara Payquerê: Centro de Educação Ambiental e Apoio à Pesquisa $\left(25^{\circ} 29^{\prime} 520^{\prime \prime} \mathrm{S}\right.$ e $49^{\circ} 39^{\prime} 243^{\prime \prime} \mathrm{W}$ ), propriedade rural de Pedro Rosa (Cerâmica Brasília), com área de aproximadamente 40 alqueires (96.000 $\mathrm{m}^{2}$ ), localizada no Distrito do Bugre, Município de Balsa Nova, Estado do Paraná, Sul do Brasil.

A área de estudo encontra-se inserida no ecótone entre os biomas da Floresta de Araucária e dos Campos Gerais, sendo o limite entre estes a Escarpa Devoniana, que divide o Primeiro do Segundo Planalto Paranaense (MAAcK 1968). A cobertura vegetal da Chácara Payquerê pode ser dividida em três partes, sendo elas: Campos Gerais, Floresta Primária Alterada e Floresta Secundária com vários estágios de regeneração. A natureza topográfica da área é de superfície altamente acidentada com inclinações de até $90^{\circ}$. A área de estudo tem sua maior altitude a $1145 \mathrm{~m}$ e a menor de $935 \mathrm{~m}$, apresentando um desnível de mais de $100 \mathrm{~m}$.

O clima é do tipo Cfb (temperado) segundo a classificação de Köppen (IAPAR 1978). A região não apresenta uma estação seca e sim uma estação chuvosa e outra menos chuvosa, possuindo uma precipitação anual média de $1600 \mathrm{~mm}$. A maior precipitação ocorre em janeiro e a menor em agosto, com médias de $304 \mathrm{~mm}$ e $77 \mathrm{~mm}$, respectivamente. A temperatura média anual fica em torno de $18^{\circ} \mathrm{C}$ (dados obtidos na estação meteorológica do Município da Lapa, Paraná).

\section{Métodos}

O estudo teve a duração de 12 meses, de fevereiro de 2002 a janeiro de 2003. Foram realizadas 20 fases de campo com duração média de três dias, obtendo-se observações diretas dos primatas em 17 delas, somando um total de 76 horas e 35 minutos de contato visual com os animais.

O grupo de estudo contava com seis indivíduos, sendo composto por dois machos adultos (MA1 e MA2), três fêmeas adultas (FA1, FA2 e FA3) e um juvenil II macho (JII) de acordo com a classificação sexo-etária proposta por MENDEs (1989). Em dezembro de 2002 houve o nascimento de dois infantes: um macho (INF1) da FA1 e uma fêmea (INF2) da FA3. A área de vida deste grupo foi predominantemente composta por floresta secundária.

Para o estudo qualitativo foram utilizados dois métodos complementares: (1) observação direta, que consistiu na mar- cação e posterior identificação das árvores onde os bugios se alimentaram; e (2) análise do conteúdo fecal, que consistiu na identificação de sementes presentes nas fezes coletadas em campo de A. guariba. Para a quantificação do tempo despendido pelo A. guariba no consumo dos diferentes itens alimentares (fruto, folha, flor e outros), foi utilizado o método de varredura instantânea (scan sampling) a cada cinco minutos (ALTMANN 1974). Foi considerado comportamento alimentar quando verificado que o animal estava de posse de algum item alimentar (folha, fruto, flor ou semente) na mão, boca ou quando estava selecionando itens ou partes a serem ingeridas.

\section{RESULTADOS}

\section{Análise qualitativa}

Os bugios utilizaram como fonte de recursos alimentares um número relativamente baixo de espécies $(\mathrm{N}=34)$. Foram observadas e marcadas 74 árvores onde os bugios se alimentaram. Neste total, foram representadas 19 famílias, das quais Lauraceae contou com seis espécies, sendo a mais representada. As análises das fezes revelaram 13 espécies consumidas (sendo sete espécies exclusivamente amostradas por este método). As espécies e os itens alimentares utilizados pelos bugios no Bugre estão representados na tabela I.

\section{Análise quantitativa}

Um total de 107 varreduras instantâneas, somando 547 registros individuais de comportamento alimentar foram registrados e analisados. Os itens alimentares foram divididos nas categorias: folha, fruto, flor e outros. A maior freqüência do período de alimentação esteve relacionada às folhas, com $57,3 \%$. Os frutos obtiveram a freqüência de $41 \%$; as flores representaram os 1,7\% restantes do tempo dedicado à alimentação pelos animais. Não havendo, portanto, outros itens observados durante as fases de campo (Fig. 1).

\section{DISCUSSÃO}

\section{Análise qualitativa}

Os bugios apresentaram como parte de sua dieta 43 itens diferentes dentre as 34 espécies consumidas. Nove espécies foram utilizadas como fontes de dois itens alimentares e 25 espécies foram fontes de um só item, mostrando uma relativa seletividade deste primata com relação a seu alimento.

Estes números parecem baixos, principalmente se comparados aos do trabalho de S. STEINMETz (com. pess.) que obteve 95 espécies na dieta de bugio-ruivo em ambiente de Floresta Ombrófila Densa no Parque Estadual Intervales em São Paulo. Chiarello (1994) reporta 68 espécies consumidas em Floresta Estacional Semidecidual em Santa Genebra, Campinas, também em São Paulo. Aguiar et al. (2003) mostram 49 espécies na dieta de bugios no Norte do Paraná, bioma de Floresta Estacional Semidecidual. Já os dados obtidos por T.P. KASECKER (com. pess.) em Floresta de Araucária são bem próximos aos

Revista Brasileira de Zoologia 21 (4): 821-826, dezembro 200W 


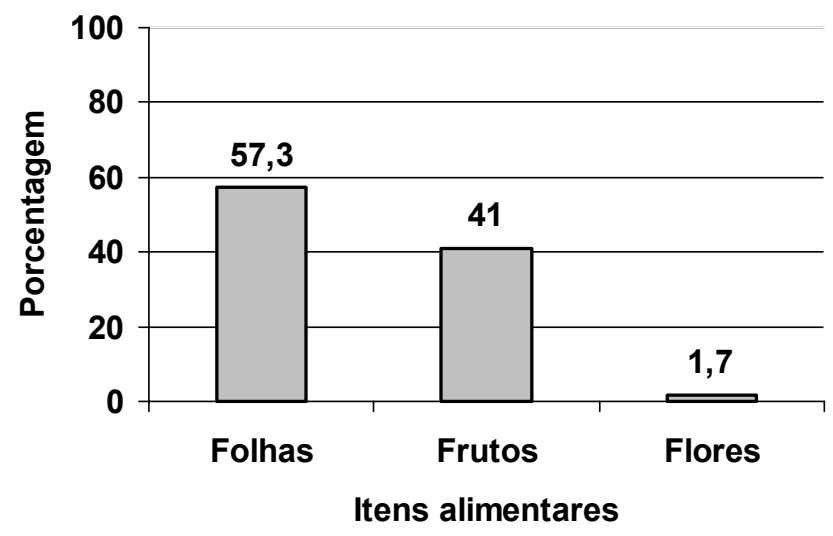

Figura 1. Proporção de tempo empregado por Alouatta guariba se alimentando dos diferentes itens $(N=547$ registros).

aqui apresentados, relatando apenas 31 espécies utilizadas pelos bugios.

Esta discrepância em relação ao número de espécies é diretamente proporcional à riqueza encontrada nestes diferentes tipos florestais, onde a Floresta Ombrófila Densa é a mais rica e a Floresta de Araucária a mais pobre, sendo a Floresta Estacional Semidecidual intermediária em número de espécies presentes (RODERJAN et al. 1998).

Houve um grande consumo de alimentos, principalmente frutos e de plantas exóticas ao ambiente de Floresta de Araucária no período entre maio a agosto, tais como Eryobothria japonica (Thunb.) Lindl. (ameixa-amarela) e Diospyrus kaki L. F. (caqui), mostrando uma boa capacidade deste primata em se adaptar a ambientes moderadamente alterados como é o caso da área ocupada pelo grupo de estudo. Bicca-Marques \& Calegaro-MarQUES (1994a) estudando $A$. caraya, mostram a importância das espécies exóticas na alimentação do bugio-preto. Silveira \& Codenotti (2001) também reportam o consumo de Diospyrus kaki para A. guariba no Rio Grande do Sul. Além da utilização de espécies exóticas, o alto consumo de folhas de Mimosa scabrella Benth. (bracatinga) e Gochnatia polymorpha (Less.) Cabr. (cambará) durante o inverno também mostra a plasticidade adaptativa dos bugios-ruivos. Isto porque estas plantas são consideradas espécies pioneiras e se mostram bem presentes em vários estágios sucessionais da Floresta de Araucária e outras formações florestais comuns no Sul do Brasil.

Em outros trabalhos com a dieta das diferentes espécies do gênero Alouatta, algumas plantas estão freqüentemente presentes e geralmente são consideradas como espécies importantes na dieta desses primatas. Estas, geralmente, são espécies que frutificam várias vezes por ano ou são fontes de folhas durante grande parte do período. Este é o caso de: Cecropia spp., Inga spp., Ficus spp., Ocotea spp., Nectandra spp., Sorocea sp. entre outras (Milton 1980, Silva JR. 1981, Chitolina \& Sander 1981, Chiarello 1994, Bicca-Marques \& Calegaro-Marques 1994b, Silveira \& Codenotti 2001, Aguiar et al. 2003, Steinmetz, com. pess.). To- davia, no presente estudo mostraram-se importantes para a dieta de A. guariba as espécies: Ocotea porosa, O. puberulla, O. odorifera, Gochnatia polymorpha e Mimosa scabrella. Tais espécies estiveram presentes na dieta desta espécie durante grande parte do ano, servindo como importante fonte de frutos e folhas.

\section{Analise quantitativa}

Nos diversos estudos realizados com as diferentes espécies de bugios a dieta destes animais variou muito em relação à proporção dos itens e em relação às espécies consumidas. A tabela II compara os resultados deste trabalho com os de outros autores. Esta variação pode ser devida às características dos vários ambientes estudados, cujas espécies vegetais prevalentes diferem muito. Apesar da metodologia utilizada neste trabalho não ser voltada a um estudo de preferência alimentar, aparentemente os bugios tendem a procurar itens sazonais (frutos, flores e sementes) quando estes se encontram disponíveis, em detrimento às folhas. Isto também é reportado por outros autores (Milton 1980, Mendes 1989, Bicca-MarQues \& Calegaro-Marques 1995).

No presente estudo, realizado em um bioma diferente e mesmo havendo uma mudança significativa nas espécies consumidas, os bugios se mantiveram principalmente folívoros. Este predomínio de folhas na alimentação foi proposto por Milton (1980) e Bicca-Marques \& Calegaro-Marques (1995) como um padrão para o gênero Alouatta como um todo. Apenas os dados de Bonvicino (1989) com A. belzebul (Linnaeus, 1766) ficam fora deste, obtendo uma freqüência relativamente alta de frutos na dieta desta. Porém, em sua pesquisa, durante a estação chuvosa, A. belzebul alimentou-se basicamente de frutos $(91,5 \%)$, o que desvia a média anual do consumo deste item.

Observando-se a tabela II pode-se verificar que os bugios tenderam a se alimentar mais de folhas que de frutos, concordando com um padrão de folivoria como o hábito alimentar principal dos bugios (Milton 1980, Chapman 1987, Neville et al. 1988, Bonvicino 1989, Mendes 1989, Chiarello 1994, Bicca-MarQues \& Calegaro-Marques 1994b, Valle et al. 2001, Fuentes et al. 2003, Aguiar et al. 2003). Entretanto, é importante destacar que embora os bugios sejam considerados folívoros, a importância dos frutos em sua dieta pode variar de acordo com as espécies de Alouatta analisadas, em diferentes biomas e sazonalmente com os frutos podendo assumir uma maior importância em determinados períodos do ano (Chapman 1987, MENdes 1989, Strier 1992, Chiarello 1994, Queiroz 1995). Esta variação sazonal também é verificada para outros gêneros de primatas neotropicais, como por exemplo, nos estudos sobre Callithrix Erxleben, 1777 (Stevenson \& Rylands 1988, Vilela \& Faria 2002); Saguinus Hoffmannsegg, 1807 (SNOwdon \& SoInI 1988); Leontopithecus Lesson, 1840 (Passos 1999); Pithecia Desmarest, 1804 (SETZ 1994) dentre outros.

A folivoria como estratégia alimentar é fortemente associada ao tamanho do corpo do animal, que em conjunto influenciam os tamanhos de grupos e a área de vida destes primatas (Milton \& May 1976, Clutton-Brock \& Harvey 1977, Strier 1992).

Revista Brasileira de Zoologia 21 (4): 821-826, dezembro 200W 
Tabela I. Espécies utilizadas na alimentação por Alouatta guariba no período de fevereiro de 2002 a janeiro de 2003, em floresta com Araucária do Estado do Paraná

\begin{tabular}{|c|c|c|c|c|}
\hline Espécie & Nome popular & Família & Recurso & Fonte do dado \\
\hline Lithraea brasiliensis March. & Bugreiro & Anacardiaceae & Folha & Obs. direta \\
\hline Schinus therebintifolius Raddi & Arroeira & Anacardiaceae & Folha, fruto & Obs. direta \\
\hline Annona cacans Warm. & Araticum-cagão & Annonaceae & Fruto & Obs. direta + fezes \\
\hline Rollinia sylvatica (A. St. Hil.) Mart. & Araticum & Annonaceae & Fruto & Obs. direta \\
\hline Guatteria australis A. St. Hil. & Pindaíba-preta & Annonaceae & Fruto & Fezes \\
\hline Ilex paraguariensis St. Hil. & Erva-mate & Aquifoliaceae & Folha, fruto & Obs. direta + fezes \\
\hline Araucaria angustifolia (Bertol.) O. Kuntze & Pinheiro-do-Paraná & Araucariaceae & semente & Obs. direta \\
\hline Syagrus romanzofiana (Cham.) Glassman & Jerivá & Arecaceae & Fruto & Obs. direta \\
\hline Geonoma schottiana Mart. & Guaricana & Arecaceae & Fruto & Fezes \\
\hline Gochnatia polymorpha (Less.) Cabr. & Cambará & Asteraceae & Folha & Obs. direta \\
\hline Vernonia discolor (Spreng.) Less. & Vassourão-preto & Asteraceae & Folha & Obs. direta \\
\hline Piptocarpha angustifolia Dusén & Vassourão-branco & Asteraceae & Fruto & Fezes \\
\hline Clytostoma binatum (Thumb.) Sandwith & Trepadeira-roxa & Bigoniaceae & Flor, Folha & Obs. direta \\
\hline Pseudobombax marginatum (St. Hil.) A. Robyns & Paineira & Bombacaceae & Folha & Obs. direta \\
\hline Chorisia speciosa St. Hil. & Paineira & Bombacaceae & Folha & Obs. direta \\
\hline Clethra scabra Pers. & Carne-de-vaca & Clethraceae & folha & Obs. direta \\
\hline Diospyrus kaki L.f. & Caqui & Ebenaceae & Fruto, folha & Obs. direta + fezes \\
\hline Alchornea triplinervia (Spreng.) Muell. Arg. & Tapiá & Euphorbiaceae & Folha & Obs. direta \\
\hline Sebastiania commersoniana (Baill) Smith \& Downs & Branquilho & Euphorbiaceae & folha & Obs. direta \\
\hline Casearia sylvestris Swartz & Chá-de-bugre & Facourtiaceae & folha & Obs. direta \\
\hline Ocotea porosa (Ness) L. Barroso Barr. & Imbuia & Lauraceae & Folha, fruto & Obs. direta \\
\hline Ocotea puberulla (Rich.) Ness & Canela-sebo & Lauraceae & Folha, fruto & Obs. direta + fezes \\
\hline Ocotea odorifera (Vell.) Rohwer & Sassafrás & Lauraceae & Fruto & Obs. direta + fezes \\
\hline Ocotea minarum (Ness) Mez. & Canela & Lauraceae & Fruto & Fezes \\
\hline Nectandra megapotamica (Spreng.) Mez. & Canela-preta & Lauraceae & Fruto & Fezes \\
\hline Nectandra sp. & Canela & Lauraceae & Fruto & Fezes \\
\hline Cedrela fissilis Vell. & Cedro & Meliaceae & Folha & Obs. direta \\
\hline Mimosa scabrella Benth. & Bracatinga & Mimosaceae & Folha & Obs. direta \\
\hline Ficus sp. & Figueira & Moraceae & Folha, fruto & Obs. direta + fezes \\
\hline Rapanea ferruginea (Ruiz et Pav.) Mez. & - & Myrsinaceae & Folha & Obs. direta \\
\hline Eryobotrya japonica (Thunb.) Lindl. & Ameixa-amarela & Rosaceae & Fruto, folha & Obs. direta \\
\hline Prunus sellowii Koehne & Pessegueiro-bravo & Rosaceae & Fruto & Fezes \\
\hline Psidium cattleianum Sabini & Araçá-vermelho & Myrtaceae & Fruto, folha & Obs. direta \\
\hline Campomanesia xanthocarpa O. Berg. & Guabiroba & Myrtaceae & Fruto & Obs. direta \\
\hline
\end{tabular}

Em seu estudo sobre estratégias ecológicas dos Atelidae STRIER (1992) mostra que as espécies de Alouatta são as mais folívoras dentre os primatas neotropicais. A dieta baseada em folhas é de baixo conteúdo calórico, o que pode influenciar toda a ecologia e o comportamento dos bugios, como, por exemplo, diminuindo as áreas de vida, os percursos diários e o período de atividades em relação a outros de mesmo porte e pertencentes a outras categorias tróficas (Milton 1980, STRIER 1992).

Apesar de os bugios apresentarem uma dieta variada, as espécies do gênero Ocotea podem ser consideradas como um importante recurso alimentar, neste remanescente de Floresta de Araucária. Mesmo sendo o presente estudo feito em um tipo florestal diferente dos mais comumente estudados, o bugio-ruivo manteve o padrão de folivoria. Alouatta guariba mostrou-se bem adaptado a uma floresta secundária, caracterizada por um dossel baixo (aproximadamente 6-8 m), alimentando-se de espécies pioneiras, como Mimosa scabrella e Gochnatia polymorpha e espécies exóticas, como Diospyrus kaki e Eryobothrya japonica.

Revista Brasileira de Zoologia 21 (4): 821-826, dezembro 200W 
Tabela II. Comparação do percentual de utilização de folhas, frutos e flores por Alouatta spp. em diferentes estudos.

\begin{tabular}{|c|c|c|c|c|c|}
\hline Espécie & Folhas & Frutos & Flores & Local & Autor (ano) \\
\hline A. guariba & 57,3 & 41,0 & 1,70 & Bugre: PR/BR & Presente estudo \\
\hline A. guariba & 50,3 & 47,9 & 1,30 & Ibiporã: PR/BR & Aguiar et al. (2003) \\
\hline A. guariba & 70,6 & 29,4 & - & SP/BR & SILVA JR. (1981) \\
\hline A. guariba & 70,6 & 15,6 & 8,35 & MG/BR & Mendes (1989) \\
\hline A. guariba & 73,0 & 5,0 & 12,00 & $\mathrm{SP} / \mathrm{BR}$ & С HIARELO (1994) \\
\hline A. belzebul & 13,3 & 59,0 & 27,60 & $\mathrm{~PB} / \mathrm{BR}$ & BonVICINO (1989) \\
\hline A. seniculus & 45,5 & 47,3 & 1,50 & AM/BR & Queroz (1995) \\
\hline A. caraya & 60,9 & 28,9 & 2,70 & $\mathrm{RS} / \mathrm{BR}$ & Bicca-M ARQues \& CALEGARO-M ARQues (1994b) \\
\hline A. palliata & 63,6 & 12,2 & 18,50 & Panamá: Barro Colorado & Neviue et al. (1988) \\
\hline A. palliata & 48,2 & 42,1 & 9,60 & Panamá: Barro Colorado & MiLTON (1980) \\
\hline A. palliata & 49,0 & 28,5 & 22,50 & Costa Rica & ChAPMAN (1987) \\
\hline A. palliata & 72,0 & 14,0 & 13,00 & México: Tabasco & VAШE et al. (2001) \\
\hline A. palliata & 76,0 & 19,0 & 5,00 & México: Tabasco & Fuentes et al. (2003) \\
\hline
\end{tabular}

\section{AGRADECIMENTOS}

Os autores agradecem ao Biólogo Édner Rosa, pelo apoio e pela permissão de realizar este trabal ho em sua propriedade; ao Biólogo Kauê C. Abreu e ao acadêmico Itiberê P. Bernardi pelo auxílio em campo.

\section{REFERÊNCIAS}

Aguiar, L.M.; N.R. Reis; G. Ludwig. \& V.J. Rocha. 2003. Dieta, área de vida, vocalizações e estimativas populacionais de Alouatta guariba em um remanescente florestal no Norte do estado do Paraná. Neotropical Primates, Washington, 11 (2): 78-86.

Altmann, J. 1974. Observational study of behavior: sampling methods. Behavior, Leiden, 40: 227-267.

Aurıcchio, P. 1995. Primatas do Brasil. São Paulo, Terra Brasilis, $168 \mathrm{p}$.

Bicca-Marques, J.C. \& C. Calegaro-M arques. 1994a. Exotic plant species can serve as staple food sources for wild howler populations. Folia Primatologica, Basel, 63: 209-211.

Bicca-M arques, J.C. \& C. Calegaro-Marques. 1994b. Feeding behavior of the Black Howler Monkey (Alouatta caraya) in a seminatural forest. Acta Biologica Leopoldensia, São Leopoldo, 1 (2): 64-84.

. 1995. Ecologia alimentar do Gênero Alouatta Lacépède, 1799 (Primates, Cebidae). Ciência Agronômica, Cadernos UFAC, Rio Branco, 03: 23-49.

Bonvicino, C.R. 1989. Ecologia e comportamento de Alouatta belzebul (Primates: Cebidae) na mata atlântica. Revista Nordestina de Biologia, João Pessoa, 6 (2): 149-179.

Cabrera, A. 1957. Catalogo de los Mamiferos de America del
Sur. Museo Argentino de Ciencias Naturales Bernardino Rivadavia e Instituto Nacional de Investigación de las Ciencias Natural es, Buenos Aires, 4 (1): 01-627.

Chapman, C.A. 1987. Flexibility in diets of three species of Costa Rican Primates. Folia Primatologica, Basel (Switzerland), 49: 90-105.

Chiarello, A.G. 1994. Diet of the Brown Howler Monkey Alouatta fusca in a semi-deciduous Forest fragment of Southeastern Brazil. Primates, Tokyo, 35 (1): 25-34.

Chitolina, O.P. \& M. SAnder. 1981. Contribuição ao conhecimento da al imentação de Alouatta guariba clamitans Cabrera,1940 em hábitat natural no Rio Grande do Sul (Cebidae, Alouattinae). Iheringia, Porto Alegre, 59: 37-44.

Clutton-Brock, T.H. \& P.H. Harvey. 1977. Primate ecology and social organization. Journal of Zoology, London, 183: 139.

Emmons, L.H. \& F. Feer. 1997. Neotropical Rainforest Mammals. Chicago, University of Chicago Press, XVI+307p.

Fuentes, E.; A. Estrada; B. Franco; M. Magaña; Y. Decena; D. Muñoz \& Y. GARCIA. 2003. Reporte preliminar sobre el uso de recursos al imenticios por una tropa de monos aulladores, Alouatta palliata, en el Parque La Venta, Tabasco, México. Neotropical Primates, Washington, 11 (1):24-29.

IAPAR. 1978. Cartas climáticas básicas do Estado do Paraná. Londrina, Instituto Agronômico do Paraná, 38p.

IBGE. 1992. Manual Técnico da Vegetação Brasileira. Manuais técnicos em geociências. Rio de Janeiro, IBGE, 166p.

LANGE, R.B. \& E.F. JABLONSKı. 1981. Lista prévia dos Mammalia do Paraná. Estudos de Biologia, Curitiba, 6: 1-35.

MAACK, R. 1968. Geografia física do Estado do Paraná. Curitiba, Max Roesner, 350p. 
Margarido, T.C. \& R. Lange. 1995. Espécies de Mamíferos ameaçadas de extinção no Paraná, p. 5-45. In: SEMA/GTZ (Eds). Lista Vermelha de animais ameaçados de extinção no Estado do Paraná. Curitiba, SEMA/GTZ, 177p.

Margarido, T.C.C. \& F.G. Braga. 2004. Mamíferos, p. 27-142. In: S.B. Mickich \& R.S. BéRnils. (Eds). Livro vermelho da fauna ameaçada no estado do Paraná. Curitiba, Governo do Estado do Paraná, IAP, SEMA, 763p.

Mendes, S.L. 1989. Estudo ecológico de Alouatta fusca (Primates: Cebidae) na Estação Biológica de Caratinga, MG. Revista Nordestina de Biologia, João Pessoa, 6 (2): 71-104.

Milton, K. 1980. The foraging strategy of Howler Monkeys a study in Primate economics. New York, Columbia University Press, XVI+165p.

Mitton, K. \& M.L. May. 1976. Body weight, diet and home range area in primates. Nature, London, 259: 459-462.

Neville, M.K.; K.E. Glander; F. Braza \& A.B. Rylands. 1988. The Howling Monkeys, Genus Alouatta, p. 349-453. In: R.A. Mittermeier; A.B. Rylands; A. Coimbra-Filho \& G.A.B. Fonseca (Eds). Ecology and Behavior of Neotropical Primates. Washington, World Wildlife Fund, vol. 2, 610p.

NowaK, R.M. 1999. Primates of the world. Baltimore, John Hopkins University Press, 224p.

Passos, F.C. 1999. Dieta de um grupo de mico-leão-preto, Leontopithecus chrysopygus (Mikan) (Mammalia, Callitrichidae), na Estação Ecológica dos Caetetus, São Paulo. Revista Brasileira de Zoologia, Curitiba, 16 (Supl.1): 269-278.

Queiroz, H.L. 1995. Preguiças e guaribas. Os mamíferos folívoros arborícolas do Mamirauá. Rio de Janeiro, Sociedade Civil Mamirauá, MCT, CNPq, XII+161p..

Redford, K.H. \& J.F. Eisenberg. 1999. Mammals of the Neotropics. The Central Neotropicas. Ecuador, Peru, Bolivia, Brazil. Chicago, University of Chicago Press, vol.
3, X+609p.

Roderjan, C.V.; Y.S. Kunyoshi \& F. Galvão. 1998. As regiões fitogeográficas do Paraná. Acta Florestalia Brasiliensis, Curitiba, 1: 1-5.

SETZ, E. 1994. Feeding ecology of golden-faced sakis. Neotropical Primates, Washington, 2 (4): 13-14.

SiLva JR., E.C.J. 1981. A preliminary survey of Brown howler monkeys (Alouatta fusca) at the cantareira Reserve (São Paulo, Brazil). Revista Brasileira de Biologia, Rio de Janeiro, 41 (4): 897-909.

Silveira, R.M.M. \& T.L. CodenotTr. 2001. Interações sociais e dieta do bugio-ruivo, Alouatta guariba clamitans, no Parque Estadual de Itapuã, Rio Grande do Sul, Brasil. Neotropical Primates, Washington, 9 (1): 15-19.

Snowdon, C.T. \& P. Solns. 1988. The Tamarins, Genus Saguinus, p. 223-298. In: R.A. Mittermeier; A.B. Rylands; A. CoimbraFilho \& G.A.B. Fonseca (Eds). Ecology and Behavior of Neotropical Primates. Washington, World Wildlife Fund, vol. 2, 610p.

Stevenson, M.F. \& A.B. Rylands. 1988. The Marmosets, Genus Callthrix, p. 131-222. In: R.A. Mittermeier; A.B. Rylands; A. Coimbra-Filho \& G.A.B. Fonseca (Eds). Ecology and Behavior of Neotropical Primates. Washington, World Wildlife Fund, vol. 2, 610p.

STRIER, K.B. 1992. Atelinae adaptations: behavioral strategies and ecological constraints. American Journal of Physical Anthropology, Columbus, 88: 515-524.

Valle, Y.G.; D. Muñoz; M. Magaña-Alejandro; A. Estrada \& B. Franco. 2001. Uso de plantas como alimento por monos aulladores, Alouatta palliata, en el Parque Yumká, Tabasco, México. Neotropical Primates, Washington, 9 (3): 112-118.

VILela, S. L. \& D. S. Faria. 2002. Dieta do Callithrix penicillata (Primates, Callitrichidae) em área de cerrado no Distrito Federal, Brasil. Neotropical Primates, Washington, 10 (1):17-20.

Recebido em 15.III.2004; aceito em 31.X.2004. 